\section{Regards sur l'économie allemande}

Bulletin économique du CIRAC

$81 \mid 2007$

Varia

\title{
Mobilité et multifonctionnalité séduisent les
} Allemands

Isabelle Bourgeois et Solène Hazouard

\section{CpenEdition}

Édition électronique

URL : http://journals.openedition.org/rea/658

DOI : $10.4000 /$ rea. 658

ISBN : 978-2-8218-0858-4

ISSN : 1965-0787

Éditeur

CIRAC

Édition imprimée

Date de publication : 1 mai 2007

Pagination : 23-26

ISSN : 1156-8992

Référence électronique

Isabelle Bourgeois et Solène Hazouard, " Mobilité et multifonctionnalité séduisent les Allemands », Regards sur l'économie allemande [En ligne], 81 | mai 2007, document 3, mis en ligne le 01 mai 2009, consulté le 30 avril 2019. URL : http://journals.openedition.org/rea/658 ; DOI : 10.4000/rea.658 


\title{
Mobilité et multifonctionnalité séduisent les Allemands
}

\author{
Isabelle Bourgeois et Solène Hazouard
}

Le volume du marché ouest-européen de l'électronique de loisirs s'est chiffré à 59,6 milliards € en 2006 selon le rapport de l'EITO publié en février 2007. La part de l'Allemagne est de $22 \%$, ce qui la situe au second rang après la GrandeBretagne (25\%), la France étant $3^{e}(17 \%)$. Ce palmarès semble stable. Selon l'institut GfK qui prend en compte d'autres catégories de produits que l'EITO et considère l'UE 15, l'Allemagne occupait en 2005 le second rang derrière la Grande-Bretagne et devant la France avec 19,5\% d'un marché estimé à 39,8 milliards $€$. Il est vrai que, en comparaison des autres Européens, les Allemands sont particulièrement bien équipés, notamment dans le haut de gamme, et que c'est principalement la tendance à la substitution qui tire le marché, d'autant que les prix des appareils de nouvelle génération ont considérablement baissé.

En 2006, la croissance du marché ouest-européen ( $+9 \%$ en moyenne, $+8,2 \%$ en Allemagne, $+10,8 \%$ en France et $+12 \%$ en Grande-Bretagne ; EITO) est largement imputable d'une part à la vente d'écrans plats, dopée par la Coupe du Monde de football. L'événement s'est traduit, plus généralement, par un record des ventes de biens d'équipement durables outre-Rhin, stimulée au dernier trimestre 2006 par l'anticipation de la hausse de la TVA, passée à $19 \%$ en janvier de cette année. D'autre part, tout comme leurs voisins européens, les Allemands s'équipent massivement en systèmes de navigation GPS, mobilité oblige. Si l'électronique de loisirs est un marché de substitution caractérisé, les technologies de l'information et les télécommunications commencent à s'approcher du niveau de la saturation. Et si une certaine convergence s'esquisse au plan des équipements, l'offre de contenus, encore embryonnaire, bride l'essor du marché.

L'électronique de loisirs 'pèse' $60 \%$ du marché de l'électronique grand public. Le segment est tiré outre-Rhin par la vente d'écrans plats (3 millions d'unités en $2006 ; 2,7$ millions de tubes cathodiques), un succès lié au Mondial de football seIon la Gesellschaft für Unterhaltungs- und Kommunikationselektronik (GfU),. La Fédération des entreprises de l'économie de l'information BITKOM, pour sa part, estime que les 4 millions seront franchis en 2007 - sous l'effet de la baisse des prix que souligne la GfU. Dans le segment des écrans de télévision, qui représente $37 \%$ du marché de l'électronique de loisirs (2005, GfK et GfU), les écrans LCD et plasma dominent avec plus de $80 \%$ de parts de marché (2006, GfU). La préférence va aux grands écrans, au format $16 / 9$ : près de $78 \%$ du CA des téléviseurs LCD revient aux écrans de $80 \mathrm{~cm}$ ( $\geq 32$ pouces) ou plus. Les Allemands n'hésitent pas à payer le prix du confort et de la modernité : en 2006, ils ont dépensé en moyenne $767 €$ pour leur téléviseur, 25 \% de plus qu'en 2005.

Evolution du volume des ventes de l'électronique de loisirs en RFA, 2005/03

\begin{tabular}{|lr||lr|}
\hline Téléviseurs à tube cathodique & $-17 \%$ & Enregistreurs de DVD & $+388 \%$ \\
Téléviseurs LCD & $+611 \%$ & Terminaux satellite & $+22 \%$ \\
Téléviseurs plasma & $+872 \%$ & Terminaux câble & $+1413 \%$ \\
Magnétoscopes & $-60 \%$ & Terminaux TNT & $+929 \%$ \\
Appareils photo numériques & $+63 \%$ & Lecteurs MP3 & $+572 \%$ \\
Lecteurs de DVD & $-25 \%$ & & \\
\hline
\end{tabular}

Source des données : Deutsche Bank Research, IT, Telekom \& neue Medien : Am Beginn der technologischen Konvergenz, 30-03-2006 (www.dbresearch.de).

Ecrans LCD et plasma : $80 \%$ du marché des téléviseurs 


\section{Marché de l'électronique grand public : 3 catégories}

Selon l'Institut GfK et la société GFU, le marché de l'électronique grand public comprend l'électronique de loisirs, les technologies de l'information et les télécommunications. L'électronique de loisirs regroupe les appareils audio et vidéo, les appareils photo numériques, les boîtiers câble, satellite et TNT et les antennes satellite (hors Pay-TV), les supports enregistrables, les jeux vidéo (consoles et logiciels) et les accessoires (écouteurs, télécommandes multifonctions). Dans la catégorie des technologies de l'information, on trouve les ordinateurs (PC et portables), les périphériques comme les moniteurs, imprimantes et PDA, les scanners et les cartes PC-télévision. Enfin, les télécommunications incluent les téléphones fixes, les portables ainsi que les fax. GfK et GFU ont réuni ces trois catégories au sein d'une même étude (Consumer electronics) du fait de la tendance à la convergence.

Les JO de Pékin lanceront la TVHD outre-Rhin

Forte demande de systèmes Dolby digital

Vidéo HD : le problème de la confrontation Blu Ray/HD DVD

$14 \%$ des Allemands possèdent une console de jeux

Net engouement pour les lecteurs MP3, les clés USB...
En 2006, $70 \%$ des écrans LCD et plasma vendus (2 millions d'unités), étaient compatibles « HD ». La BITKOM estime que 2,5 millions de ménages sont déjà équipés pour recevoir la télévision haute définition. La fédération mise sur 5 millions fin 2007 et 17 millions d'ici à la fin 2011. Actuellement, la haute définition reste confinée à la télévision à péage. La réception de programmes de meilleure qualité visuelle implique une connexion numérique : $31,6 \%$ des ménages en disposent via une antenne DBV-T (la TNT allemande), le câble ou le satellite, soit une hausse de $6 \%$ par rapport à 2005, comme le précise le rapport Digitalisierungsbericht 2006 des instances de régulation des médias (Landesmedienanstalten). Selon la fédération de l'électrotechnique ZVEI, près de 80000 boîtiers TNT, câble et satellite HD ont été vendus l'an passé ; elle table sur 4 millions d'ici 2010. A cela viendra s'ajouter l'offre HD de la télévision par Internet. L'offre de contenus HD de TV gratuite sera lancée dès l'an prochain, avec la retransmission des JO de Pékin ; les chaînes du service public la développeront à partir de 2009.

La numérisation accroît les exigences en termes de qualité sonore, surtout pour des consommateurs habitués à la stéréophonie en radio et TV depuis plus de 30 ans. Les systèmes audio de home cinema ont ainsi permis d'enrayer la chute du marché, saturé, de la $\mathrm{Hi}-\mathrm{Fi}$ ( $-11,5 \%$ en $2005 ;-3,2 \%$ en 2006 ; CEMIX). Mais la hausse du CA $(+2,5 \%)$ ne traduit pas une augmentation des ventes (en baisse de $18,8 \%)$; elle est due à une forte augmentation des prix (+26,3\%) liée, selon I'EITO, à une demande croissante de systèmes Dolby digital de haute qualité.

Le taux d'équipement en appareils photo numériques étant déjà très élevé (42\%; Destatis), les Allemands cherchent aujourd'hui la qualité : sur les 8 millions d'appareils vendus en $2006(+5,7 \%$ en un an ; CEMIX), on dénombrait 550000 reflex numériques (GfU) - tendance à la hausse. De même, la perspective de la haute définition a profité aux caméras vidéo HD : 18000 unités ont été vendues l'an dernier (ZVEI). La hausse des performances et la miniaturisation des caméras (avec puce carte-mémoire, disque dur intégré, etc.) devrait se solder par la vente de 700000 unités à l'horizon 2010. Le salon IFA de Berlin (31-08/5-09-07) sera l'occasion de la confrontation des technologies Blu Ray (Sony et Apple) et HD DVD (Toshiba et Microsoft) présentes sur le marché depuis la fin 2006. L'enjeu est de taille : selon Destatis, $59 \%$ des ménages possédaient un lecteur/enregistreur DVD au début 2006 (contre $27 \%$ en 2003). La ZVEI mise sur la vente de 3,3 millions de lecteurs/enregistreurs de DVD haute définition d'ici à 2010. Mais la guerre des standards pourrait retarder leur diffusion, à l'instar de ce qu'on a pu observer au début des années 1980 dans le secteur de la vidéo où s'affrontaient le VHS, le Betamax de Sony et le V2000 de Philips. En attendant, Sony mise sur sa console PS3, équipée d'un lecteur Blu-ray.

Les consoles de jeux vidéo se portent plutôt bien $(+43,5 \%$ en $2005 ;+30 \%$ en 2006 ; CEMIX) à en croire Destatis qui en a recensé pour la première fois en 2006 le taux d'équipement (14\%). Les nouvelles consoles portables Sony PSP et Nintendo DS et la diversification des jeux ont profondément modifié la structure des consommateurs. Si on ne comptait en 2002 que $27 \%$ d'utilisatrices de jeux vidéo, elles sont désormais $40 \%$. Et même si $50 \%$ des 'mordus' (hommes et femmes) appartiennent à la tranche des 10-15 ans, les $20-29$ ans (+8 \%) et les plus de 40 ans $(+3 \%)$ font preuve d'un intérêt croissant (GfK).

La mobilité, outre-Rhin plus encore qu'ailleurs, pousse les ménages à acheter lecteurs MP3, clés USB et GPS. Après le boom de $2005(+125 \%$ de CA $)$, le 
segment des MP3 souffre d'un léger recul en 2006 (-2,5\% ; CEMIX). Le marché est pourtant loin d'être saturé : début 2006, $23 \%$ seulement des foyers avaient un lecteur MP3 ; les ménages au sein desquels la personne qui rapporte le revenu principal a entre 25 et 55 ans étant les mieux équipés (de $34 \%$ à $36 \%$; Destatis). Le volume des ventes de clés USB a augmenté, quant à lui, de $57 \%$ en 2005 comme en 2006. A cause des baisses de prix successives, l'évolution du $\mathrm{CA}$ a néanmoins été moins rapide $(+28,1 \%$ et $+16,5 \%$; CEMIX).

Croissance des systèmes de navigation amovibles en 2006 en Europe de l'Ouest ${ }^{\text {a) }}$

\begin{tabular}{|c|c|c|}
\hline & Nombre d'unités vendues & Variation par rapport à 2005 \\
\hline Allemagne & 2.000 .000 & $+300 \%$ \\
\hline Grande-Bretagne & 1.600 .000 & $+105 \%$ \\
\hline France & 1.230 .000 & $+290 \%$ \\
\hline Italie & 760.000 & $+245 \%$ \\
\hline Espagne & 688.000 & $+475 \%$ \\
\hline Pays-Bas & 480.000 & $+210 \%$ \\
\hline Belgique & 315.000 & $+170 \%$ \\
\hline Suède & 125.000 & $+40 \%$ \\
\hline Autriche & 110.000 & $+225 \%$ \\
\hline Suisse ${ }^{b)}$ & 95.000 & $+116 \%$ \\
\hline
\end{tabular}

Source : GfK Marketing Services Deutschland, 2007. a) Etat à la fin décembre 2006 ; b) état à la fin novembre 2006. Total des ventes : 7,4 millions; prévisions $2007: 11,8$ millions (www.gfk.de).

Quant au segment du GPS, il continue de battre tous les records. L'année 2005 s'était déjà soldée par une hausse de CA de $85 \%$, et ce malgré une chute des prix de l'ordre de $30 \%$. Le nombre de GPS vendus a plus que triplé l'an dernier (CEMIX). Les données de l'institut GfK révèlent une certaine inclination des Allemands pour le confort automobile. Si dans la plupart des segments de l'électronique de loisirs, l'Allemagne se situe au second rang derrière la GrandeBretagne, voire au troisième (la France précède l'Allemagne pour les lecteurs DVD et les caméras vidéo), elle se retrouve en tête pour les systèmes $\mathrm{Hi}-\mathrm{Fi}$ automobiles (23,4 \% de parts de marché) ainsi que pour les systèmes de navigation (32,4\%), où elle devance la Grande-Bretagne de plus de 20 points.

La catégorie des technologies de l'information et de la communication 'pèse' $40 \%$ du marché de l'électronique grand public. Après une stagnation en 2005, ce segment a subi un léger recul l'an dernier $(-3,9 \%$ pour les technologies de l'information ; $-1,6 \%$ pour les télécommunications ; CEMIX). Aujourd'hui, le taux d'équipement en téléphonie mobile a atteint les $100 \%$ en Allemagne; la tendance va nettement au multi-équipement. Près de 21 millions de portables ont été vendus l'an dernier, grâce à l'apparition de nouvelles technologies : intégration d'appareils photos de meilleure qualité, de lecteurs MP3 et d'une énorme capacité de stockage. A eux seuls, les Smartphones, ces portables doublés d'un PDA, voire d'un GPS et qui permettent l'envoi d'e-mails (750 000 unités vendues), ont réalisé un CA de 199 millions $€$ (GfU).

Au sein des TIC, les produits phares sont ceux qui répondent au besoin de mobilité des Allemands: ordinateurs portables $(+6,6 \%$ de CA en 2006) et PDA $(+10,8 \% ;$ CEMIX). Le taux d'équipement en portables s'est hissé à $21 \%$ début 2006 (17\% début 2005 ; Destatis). A l'heure actuelle, ceux-ci n'ont plus rien à envier aux PC : ils sont dotés de processeurs rapides et performants, d'une grande capacité de stockage sur le disque dur, d'applications et de connexions multifonctionnelles, et offrent en prime l'avantage de dimensions réduites. L'effet de substitution par rapport aux PC de bureau $(-15,2 \%$ en 2006) est flagrant. Les écrans plats ont là aussi pratiquement remplacé leurs ancêtres cathodiques : 3,1 millions d'écrans LCD ont été vendus en 2006 pour seulement 50000 écrans traditionnels. La préférence pour la multifonctionnalité affecte également les périphériques; I'an passé, on a vu chuter de $15 \%$ à $20 \%$ le chiffre d'affaires généré par les imprimantes, fax et scanners, et ce au profit d'appareils multifonctions (+10,5\% de CA en 2006 ; CEMIX). Cela étant, le recul du fax s'explique bien plus encore par l'évolution des modes de communication liée à la diffusion
... et surtout le GPS

La multifonctionnalité dope les ventes de téléphones portables ...

... et l'impératif de mobilité celle des ordinateurs portables 
L'avenir des équipements multifonctions dépendra de l'offre de contenus

Deux standards en concurrence pour la TV mobile
d'Internet : L'Allemagne comptait $65 \%$ d'Internautes au premier trimestre 2006 ; $85 \%$ d'entre eux se connectent pour envoyer/recevoir des e-mails (Destatis).

L'évolution des cartes PC-TV (+17,1 \% en 2006) révèle la disposition des Allemands à entrer dans l'ère de la convergence. Certes, l'IPTV et, plus généralement, le «triple play », offre combinée de téléphonie, télévision et Internet, n'en sont encore qu'à leurs débuts outre-Rhin (voir REA 78/06), mais la baisse des tarifs d'accès illimités à Internet et la progression du haut débit devraient pousser la demande. Reste la question-clef : si les opérateurs du câble et du téléphone modernisent leurs réseaux (Deutsche Telekom s'est même vu accorder le monopole du très haut débit V-DSL), ils devront aussi se concentrer sur l'élargissement de leur offre, grâce à des partenariats avec des chaînes de télévision ou des éditeurs/propriétaires de droits, afin de proposer à leurs abonnés l'accès aux contenus d'IPTV ou de vidéo à la demande. Or le défi commercial consiste à convaincre les consommateurs de la valeur ajoutée des offres payantes dans un pays où, contrairement à la France, une offre pléthorique de contenus gratuits (offre standard : 30 chaînes) avait retardé le lancement du Pay-TV. Selon l'étude BITKOM sur l'avenir de l'économie numérique (Zukunft Digitale Wirtschaft), l'Allemagne devrait combler son retard d'ici 2010 : près de 2,2 millions de foyers devraient alors utiliser l'IPTV (50 000 actuellement). En Europe, la France est en position de leader dans ce domaine : elle regroupe à elle seule la moitié des utilisateurs européens - il est vrai dans un contexte de pénurie d'offre gratuite.

Un second défi réside dans l'avenir de la télévision mobile que les Allemands ont pu découvrir pendant le Mondial de football 2006. Alexander Röder, président de I'Institut pour les technologies de l'information (ITG) au sein de la Fédération des technologies électrotechniques, de l'électronique et de l'information (VDE), et président technique de l'opérateur de téléphonie 02 , prévoit une explosion du marché à partir de 2009. Certes, des tests ont été effectués l'an passé, mais les difficultés techniques liées aux réseaux et terminaux persistent. II manque surtout à l'Allemagne un standard unique : les technologies DMB et DVB-H sont actuellement en concurrence. Restera ensuite à convaincre les téléspectateurs...

En Allemagne, les eVolutions technologiques de la dernière décennie dans l'électronique grand public ont été plutôt bien accueillies par les consommateurs qui exigent toujours plus de qualité, de mobilité et de multifonctionnalité et semblent ainsi prêts à franchir le pas de la convergence. Le salon CeBIT de Hanovre, qui se consacrait à l'origine aux seules technologies de l'information, s'est ouvert aux télécommunications et, plus récemment, à l'électronique de loisirs, où il se trouve dorénavant en concurrence avec le Consumer Electronics Show de Las Vegas et le salon IFA de Berlin, désormais annuel. L'an passé, le président de la division allemande de Samsung avait même lancé l'idée d'une fusion possible du CeBIT et de l'IFA. Même si cette proposition a trouvé peu d'échos au sein de la branche, encore très fragmentée comme en atteste aussi la multiplicité des organisations, elle en préfigure la convergence future. Pour l'instant, c'est le segment des loisirs qui tire l'électronique grand public, et plus particulièrement la TV numérique. Les Allemands sont bien équipés pour l'avenir ; ils n'attendent maintenant qu'une offre attractive de contenus, à prix abordables s'entend...

\section{Indications bibliographiques}

Arbeitsgemeinschaft der Landesmedienanstalten (ALM) et Gemeinsame Stelle DIGITALER ZUGANG (GSDZ), Digitalisierungsbericht 2006 (www.alm.de)

BITKOM, Zukunft Digitale Wirtschaft, janvier 2007 (www.bitkom.de)

EUROPEAN INFORMATION TECHNOLOGy OBSERVATORY, EITO 2007. Part Two, The CE market in Western Europe

GFK, GFU, Der Markt für Consumer Electronics - Deutschland 2005 (www.gfu.de)

GFK, Consumer Electronics Market Europe (EC) 2005 (données GfK sur le site GfU)

GFU, BVT, GFK, Consumer Electronics Marktindex Deutschland (CEMIX) : Januar 2005 Dezember 2005 \& Januar 2006 - Dezember 2006 (www.gfu.de)

www.vde.de ; www.zvei.de. 